\title{
PROTON-MICROWAVE THERAPY
}

\author{
G. Oksuzyan ${ }^{\dagger}$, M. Ivanyan, YerPhI, Yerevan, Armenia
}

\section{Abstract}

The new method of tumor therapy is proposed: joining up of proton (photon) and microwave methods. The combined tumor therapy gives the possibility of simultaneous influence on the albumen molecules (tissue cells) in vivo of $\mathrm{RF} /$ Microwave and proton (photon) radiation. Such an influence must be made not only synchronous but coherent as well. In this case the effectiveness will depend on the relative phase between the proton bunches sequence or photons, radiated tissue and radio frequency oscillations, with the help of which this bunches were formed and accelerated in accelerator. The proposed combined proton + RF/Microwave therapy method gives the possibility of simultaneously (with proton or photon) and coherent influence on the tissue cells, in the wide microwave range, included mm-range. It may be provided by multiplication of the accelerating field frequency by $\mathrm{n}$. It is necessary to mention, that the additional MW therapy is able to decrease the proton (photon) radiation dose for patients, because of appeared centers of the devitalisation of tumor tissue may be explored due to MW action (already without proton or photon radiation), i.e. it possible to obtain the more spare therapy method. The Linac of YerPhI synchrotron at energy $75 \mathrm{MeV}$, in which it was proposed to establish the proton linear accelerator, at $12 \mathrm{MeV}$ is the likely accelerator for carryint out this method of tumor therapy.

\section{PROTON-MICROWAVE THERAPY}

A creation of Proton Therapy Complex (PTC) is proposed on the base of Yerevan Electron Synchrotron. Proton acceleration will be made in the ring of electron synchrotron from the injection energy of $12 \mathrm{MeV}$ up to the $250 \mathrm{MeV}$ energy. However the proton tumor therapy in range of middle and high energy $(70-250 \mathrm{MeV})$ will be much more effectively in case of combination of the traditional and new methods of therapy. Such a combination is used already in the world practice of the tumor therapy. The reference on the electron and photon therapy combined method is given in work [1] (see p.21). The electrons in energy range of $5-25 \mathrm{MeV}$ were used.

In case of proton therapy the energy range choosing is made more exact, because of the pronons have more hight factor of Relative Biologycal Effectivity (RBE), thus for the every case of the tumor it is necessary to choose an according voxel field for proton and tissue interaction. It is making with the help of special scattering and scanning arrangements ([1], pp. 408-414). For example, in case of not deep tumors (uveal eye melanoma, skeen tumors et al.) the accelerators with the comparatively small energy

\footnotetext{
’oksuzyan@moon.yerphi.am
}

$E \leq 80 \mathrm{MeV}$ are used (see work [1], pp. 404-405, Claterbridge Hospital, Bebington, England $E=62 \mathrm{Mev}$, operating from 1990). For high energy accelerators the bunch moderators are apples with the simultaneous forming of therapy field.

Let's consider now the means of acting electron linear accelerator of YerPhY at energy $75 \mathrm{MeV}$, in which was proposed to establish the proton linear accelerator at $12 \mathrm{MeV}$. The depth of proton running with the such energy may be calculated on the base of the date of linear energy transfer (LET) $d E / d x[\mathrm{KeV} / \mu \mathrm{m}]$. For $12 \mathrm{MeV}$ the depth of run is equal $\sim 0.2 \mathrm{~cm}$. This value for the first time is enough for the initial complex investigations and skin tumor therapy. One can mention also, that in case of necessary it is possible to establish one or two additional accelerating sections (see below) and it that way to increase the depth of protons run up to $\sim 2 \mathrm{~cm}$. The means of linear accelerator in tumor therapy are amplified because of it is a source of high power radio and Microwave radiation. The question is that in the last years for the tumor therapy the radio frequency and $\mathrm{RF} /$ Microwave methods are prevalent and these using increased in the many tumor therapy centers [3-5]. At the action of the radiation in the series of cases so called latent injuries of macromolecule are arises. In this state the molecule is still able to fermentation activity. In case of oxigen introduction, or in another case of heating, the lateral injury performs to the evidently one and brings to the loss of activity of molecule. As it follows from above, the joining up of proton and microwave methods of therapy can give a essentially positive effect. It is necessary to mention, that the additional MW therapy is able to essentially decrease the proton radiation dose for patients, because of appeared centers of devitalisation of the tumor tissue may be explored due to Microwave (MW) action, i.e. it is possible to obtain the more spare therapy method. The microwave therapy have a large possibility for its modification and corresponding coordination with the proton therapy. Such a possibility, for example, is a possibility of choosing the spectral composition of the tumor microwave radiation which coincides or closes to the absorption spectrum of molecule or to the spectrum of more great molecular compositions. It is possible also microwave influence on the tumor at the simultaneous insertion in the tissue the ions of the different medical products (electrophoresis) or clear silver ions for thermal effects amplify (increasing of the factor of absorption of microwave).

As it indicated in [2], "an important role in mechanism of the radiation injury plays the migration of the initial absorbed energy along the macromolecule. The experiments with the $\alpha$ - radiation of giant albumen molecules confirms such a migration. In this molecules in 


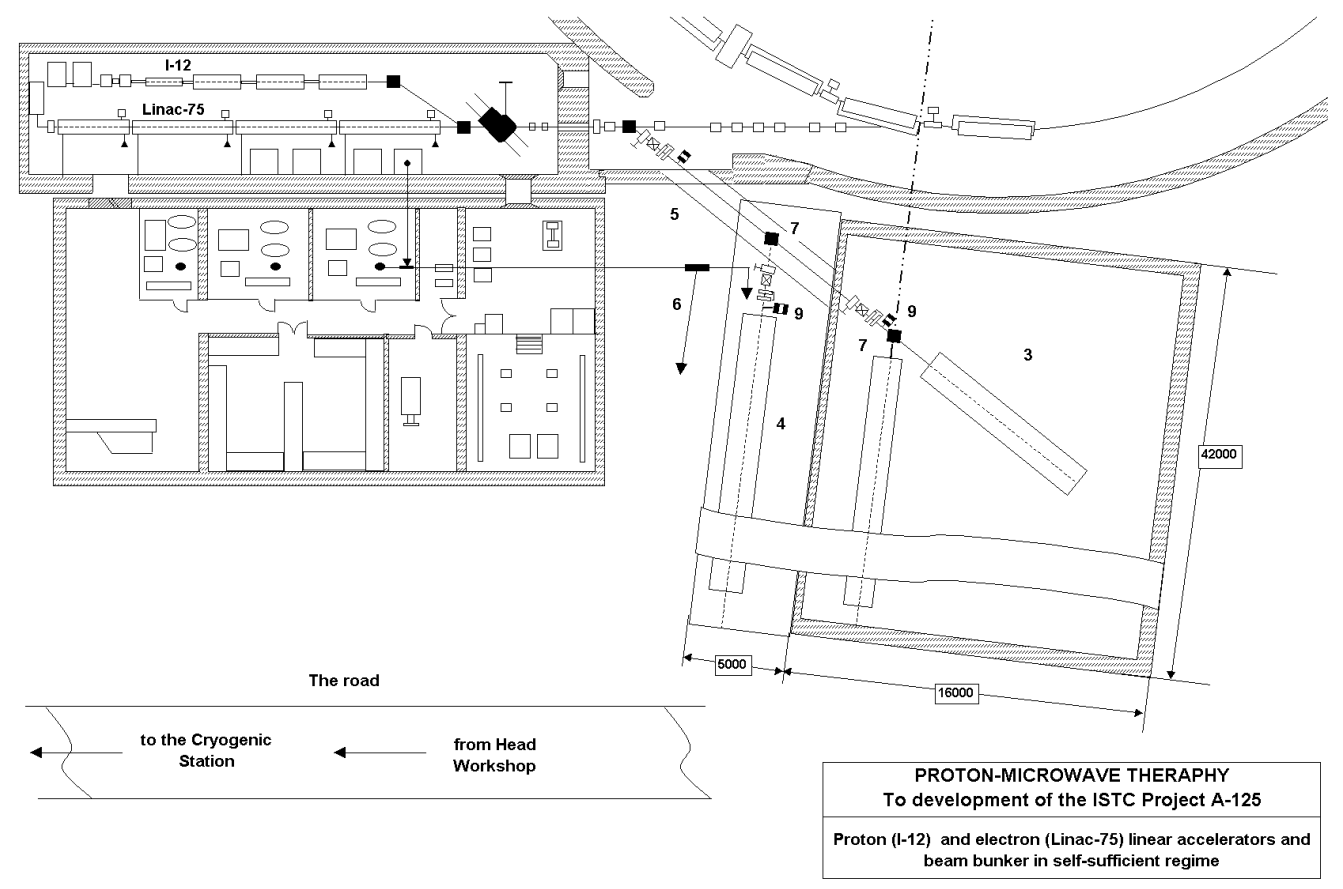

Fig.1.

main the same connections are break independently from the place of hitting of $\alpha$ - particle. The radiation injury of macromolecules becomes apparent in the loss of biological activity... in the breach and lacing appearance, in the radiation oxidation and so on.

By the help of electron spin resonance (ESR) method was established that in series of cases the lateral injury of the macromolecule is the electron excitation, accompanied with the unpaired electron appearance." It is follows from the citation, that in the microwave therapy the more thin mechanisms of influence on the tumor cell may be using in case of constant magnet field presence, i.e. to influence on the cell with the nuclear or electron spin resonance which is possible by the influence of the MW field of the determinate (resonant) frequency. Thus the basic proton tumor therapy may be, as it noticed, essentially amplified (after proton radiation) by the application of tumor microwave therapy in the spare regime for patient.

With the connection of using of the proposed combined therapy (proton $+\mathrm{RF} /$ Microwave) the next signal parameters mast be regulated: the region of generation frequency, spectral composition, the signal amplitude, uniform and impulse regimes and also the phase of RF/Microwave signal. In connect of the last parameter it is necessary to give explanation. The question is that the combined tumor therapy method gives the possibility of simultaneously influence on the albumen molecules (tissue cells) in vivo RF/Microwave and proton (photon) radiation as well. Such a influence mast be made not only synchronous but coherent as well. In this case influence effectiveness will depend from the relative phase between the proton bunches sequence or photons, radiated tissue and radio frequency oscillations, with the help of which this bunches were formed and accelerated in accelerator. It is necessary to bring here the next concept given in the work of S.P. Sitko and L.N. Mkrtchian: "If the viable embryonic cells are placed immediate close to the implanted ones, then in view of the metabolic and quantum mechanical affinity of the embryonic and cancer cells the coherent field of the $\mathrm{mm}$ range is arises between them. This is a indispensable condition of preservation of vital functions and reproduction of the cell structures." [6]. The proposed combined proton+RF/Microwave therapy method gives possibility of simultaneously (with the proton or photon) and coherent influence on the tissue cells in the wide microwave range, included $\mathrm{mm}$ - range. It may be provide by multiplication of the accelerating field frequency $\omega_{0}$ by $\mathrm{n}: \omega_{n}=n \omega_{0}$. Consequently, by the variation of frequency $\omega_{n}$ it is possible to determine the optimal (resonant) frequency of the simultaneous influence of the particle and field on the albumen structure. If one suppose that the albumen structures have properties of memory, then the continuation of tumor therapy (after influence of proton $+\mathrm{RF} /$ Microwave) with the help of Microwave therapy only (without protons) will be more effectively, than only radio frequency methods application. The memory of the albumen structures may be amplified by using of constant magnetic field with the possibility of nuclear and electron spin resonance application.

Here one can mention the specificity of $\mathrm{RF} /$ Microwave application in combination with the proton therapy: a possibility of microwave radiation of the large and small regions of organism in complex with the chemotherapy and with the magnetic field application. The microwave field uniform distribution throughout of the whole volume of the tissue let one to create an additional therapy method of such complex forms of cancer as a small-celled lung cancer, diffusive small - 
nodular cancer of liver, metastatic centers and another distributed forms of tumors. It is also possible the combination of RF/Microwave method with the proton + keylon therapy [6] and different combination of the mentioned three methods as well.

On the basis of foregoing the problem of Yerevan Electron Synchrotron application for the creating of PTC it is possible to divide on the two stage: 1) investigations and applications of the proton therapy in connection with the possibly of separate using of the beams of the synchrotron linear accelerator (proton, electron, photon, microwave), and 2) synchrotron proton beams application in energy range $70-250 \mathrm{MeV}$.

The works, carrying out at the Stage 1, will be a power base for the clinical investigations and tumor therapy and for the research engineering in the radiobiology and medicine as well, with the help of large spectrum of the nuclear and radio physical methods. On the Second Stage (in energy range $70-250 \mathrm{MeV}$ ) the methods of the word practice of the deep tumors therapy will be assimilate with application of the tumor therapy results, obtained during the complex investigations at the Synchrotron Linear Accelerator.

On Fig. 1 are brought: the location scheme of Electron Linear Accelerator (Linac-75), Proton Linear Accelerator (I-12) (1), the sections of electron injection in the Ring Accelerator (2), flat top building (3) and one-storied bunker (4), which is proposed to build for the derived bunches of the linear accelerators (for protons, electrons and photons). The canals of the derived bunches of the linear accelerators, as it was proposed before, may be placed, for example, in the flat top building basement, which area is about $700 \mathrm{~m}^{2}$ (Fig.1, (3)). However, in such a case it is necessary: an additional radiation protection, digging of the $8-\mathrm{m}$ corridor for the joining the buildings (2) and (3). In addition in this case it will be necessary to carry out the earthwork in large volume for deepening the basement down to $-1.5 \mathrm{~m}$ level. Therefore the most optimal variant for the derived bunches canal placement is a building of the one-storied bunker, the length of which is equal or less one flat top building and adjacent with one. In this case the radiation protection will be separated and at the special lateral doors placing the correspond volumes of the existing building may be used.

The radio frequency and microwave signals derivation from (1) may be realized by help of placed on the earth level waveguide-to-coaxial lines (6) with these insertion into the bunker modules or into therapy building. $\mathrm{RF} /$ Microwave signals derivation is proposed to realize from the Linac-75 klystron $\left(P_{\text {pulse }}=20 M W\right)$ hall and from the generators, feeding of the accelerator sections I12 and multiplication blocks as well.

The remaining equipment problems concerns to the $\mathrm{RF} /$ Microwave fields forming in the tissues (irradiated antennas with the corresponding patterns creating, screening, resonant conditions and so on) it is supposed to solve in the clinic and in the same place to set up an equipment with the possibility of its tuning and modification in the wide range.

As it was mentioned, the going out to this direction will give in perspective the possibility of using (through the road) an existing foundation and ferroconcrete constructions (transformer building) for the medical building creating for the purpose of clinical and scientific investigations.

The main elements of the proton and electron bunches transportation line (of the accelerators I-12 and Linac-75) to the bunker are the magnets (7), placed in the modules (1), (2) and (4).

For the normal operating of the vacuum proton tubes it is supposes to place the high vacuum ion pumps along the tracts, entered into the bunker and exiting from one. It is proposed also to establish the gates (9) and another vacuum equipment.

\section{REFERENCES}

[1] V.H. Sharf. "Biomedical Particle Accelerators. American Institute of Physics", NY, 1994.

[2] Yu..M. Shirokov, M.P.Yudin. Nuclear Physics, Moscow, "Nauka",1980 (in Russian).

[3] A. Rosen, H. D. Rosen. "RF/Microwaves in Medical Applications, and Future Research". IEEE MTT-S Newsletter, Number 148, Spring 1999.

[4] A. Winter, J. Laing, R. Paglione et al.," Microwave Hyperthermia for Brain Tumors". Neurosurgery, 17: 387, 1985.

[5] C. Rappoport, J. Pereira, "Optimal Microwave Sourse Distributions for Heating Off- Centers Tumors in Spheres of High Water Content Tissue", IEEE Trans. of Microwave Theory and Techniques, vol. 40, N10, p. 1979 , Oct. 1992.

[6] L.N.Mkrtchian. "Proton Therapy and its Peculiarities in Designed Yerevan PTK", Preprint, Yerevan, 1999.

[7] H. Wiedemann. "Particle Accelerator Physics. Basic Principles and Linear Beam Dynamics",. SpringerVerlag, 1993. 